\title{
Postcolonialisme et prise en charge institutionnelle des jeunes belgo-congolais en situation de rupture sociale (Anvers, Bruxelles)
}

\author{
Ural Manço $^{\mathrm{a}}$, Mireille-Tsheusi Robert ${ }^{\mathrm{b}}$ et Billy Kalonjic \\ a) Professeur associé, Chef du Département de sociologie, Université d'Aksaray, Turquie \\ uralmanco@aksaray.edu.tr \\ b) Présidente de l'Observatoire Ba YaYa, Bruxelles, \\ membre du Collectif Mémoire coloniale \\ mireille_robert@yahoo.fr \\ c) Responsable de la Commission socioculturelle de la Plateforme africaine d'Anvers, \\ membre de l'association Mwinda Kitoko et du Collectif Mémoire coloniale \\ billy.kalonji@samenlevingsopbouw.be
}

\begin{abstract}
Based on participant observation and the professional testimonies of two Congolese social workers in Antwerp and Brussels, this article underlines how the relationship between local authorities and Belgian-Congolese associations are reminiscent of the colonial order. These reminiscences possibly explain the dysfunctions in the social care of the Belgian-Congolese youth. The sociocultural needs of this group are not met, neither are their associations sufficiently recognized nor funded. In order to provide an explanatory basis for the 'urban gangs' phenomenon, the article also examines the youngsters' identity question by posing two hypotheses: the first concerns the breakdown of the Congolese migrant family, and the second investigates the socio-psychological impact of the colonial legacy. This approach invalidates the comparison (often made by public authorities) of the social realities of the Belgian-Congolese youth with those of young Belgians of Moroccan and Turkish descent.
\end{abstract}

Keywords

Belgium, Congolese immigration, youth policies, relation administration/migrant associations, postcolonial attitudes

\section{Résumé}

Se basant sur une observation participante et le témoignage professionnel de deux travailleurs sociaux congolais, l'article souligne, à Anvers et à Bruxelles, les rapports entre autorités locales et associations belgo-congolaises qui rappellent l'ordre colonial. Cette réminiscence peut fournir une explication aux dysfonctionnements qui apparaissent lors de la prise en charge des jeunes belgo-congolais. Les besoins socioculturels des Belgo-Congolais ne sont pas rencontrés et leurs 
associations ne sont pas assez reconnues ni financées. La contribution étudie également la question identitaire de ces jeunes en proposant deux hypothèses qui pourraient fournir une base explicative au phénomène des «bandes urbaines », concernant la déstructuration de la famille migrante congolaise et l'effet socio-psychologique des séquelles coloniales. Cette approche tend à rendre caduque la réduction (souvent opérée par les pouvoirs publics) des réalités sociales des jeunes Belgo-Congolais à celles des jeunes Belges issus des immigrations marocaine et turque.

Mots-clés

Belgique, immigration congolaise, politiques de jeunesse, rapports administration/association de migrants, attitudes postcoloniales

\section{Introduction}

C'est peu dire que la question postcoloniale fait en Belgique l'objet d'un traitement marginal, y compris dans le champ académique. Des travaux ont pourtant relevé la permanence d'une culture coloniale (Jacquemain 1985 ; Miélants 2007 ; Viaene et al. 2009) et des décalages criants entre récit national et mémoires de la colonisation (Licata and Klein 2005; Gillet 2007 ; Demart 2013a) qu'il nous semble important, pour ne pas dire urgent, d'examiner plus en profondeur. En particulier lorsque l'on se situe du point de vue de la jeunesse belge d'origine congolaise et plus généralement africaine dont certaines déviances (Lazalo Ndoma 2006 ; Lazalo Ndoma et Robert 2009 ; Mazzocchetti et Jamoule 2011) apparaissent comme le symptôme d'un malaise postcolonial touchant aux tabous de l'histoire partagée entre Belges et Congolais et à la racialisation de la société belge.

En effet, il est aujourd'hui admis que les Congolais n'eurent que de manière exceptionnelle accès au territoire de la métropole durant la colonisation en raison du besoin de main d'œuvre au Congo et de la crainte de mélange racial en colonie et donc en métropole (Etambala 1993; Kagné 2000 ; Cornet 2004). Après l'indépendance et jusqu'à la fin des années 1980, la présence des Congolais en Belgique fut caractérisée par la mobilité et de fréquents retours au Congo-Zaïre (Demart 2013b ; Kagné 2001; Tshimanga 2004; de Clercq 2002). C'est avec la sédentarisation des étudiants, consécutive à l'effondrement socioéconomique du pouvoir zaïrois, que les Congolais devinrent des «immigrés » (Lusanda 1993; Mayoyo 1995 ; Cornet 2004), au tournant des années 1990, et se confrontèrent à une véritable précarité (Lusanda op.cit.). A cette époque va alors émerger au sein de la diaspora une série d'initiatives associatives porteuses de revendications, notamment en matière de jeunesse. 
Deux décennies plus tard, force est de constater, d'une part, la marginalisation de ce secteur associatif et, d'autre part, la méconnaissance durable des institutions et pouvoirs publics vis-à-vis des résidents et des concitoyens d'origine congolaise et africaine, alors même que la Belgique détient une expertise internationale, acquise durant la colonisation, en matière congolaise.

Dans le cadre de cet article à trois voix, nous souhaitons examiner la problématique postcoloniale dans les politiques d'intégration sociale, en mettant en perspective d'un côté, les difficultés sociales et le malaise identitaire que vivent des jeunes issus de l'immigration congolaise et, par extension raciale, les jeunes d'origine africaine subsaharienne et, de l'autre, la persistance au sein des institutions étatiques de pratiques coloniales du point de vue des acteurs associatifs et militants.

Ce qui nous amènera implicitement à interroger l'absence de prise en compte des spécificités socio-culturelles et migratoires congolaises ou africaines dans la formulation et la mise en application de politiques d'intégration, pourtant elles-mêmes sujettes à un traitement différencié à la suite des émeutes maghrébines dans les années 1990 (Cartuyvels et Hebberecht 2001 Rea 2006 ; Manço et Kanmaz 2002 ; Demart 2013c).

Nous verrons que l'articulation configuration (post)coloniale/traitement racial, présente tout au long de cet article renvoie d'une part, à la situation des Congolais en Belgique qui se singularise par une présence plus importante et plus ancienne que les autres groupes originaires d'Afrique subsaharienne (Schoonvaere et Schoumaker 2013) et, bien entendu, par une histoire de plus de 150 ans avec la Belgique et, d'autre part, à la dynamique de rassemblement des personnes d'origine africaine collectivement confrontées à des discriminations d'ordre racial. Ce qui nous amènera à souvent osciller entre deux catégories, «congolais » et «africains », non pas en raison d'une imprécision de l'observation ou de la catégorie pratique mais parce que l'on a affaire dans le champ de la jeunesse ou des associations à un noyau congolais auquel des individus d'origines africaines diverses se greffent.

En l'absence de recherches approfondies sur le sujet, nous sommes partis d'expériences de travailleurs de terrain, co-auteurs de cette contribution et actifs dans le monde associatif congolais de Belgique en tant que présidente de l'association bruxelloise $B a Y a Y a$ ('Les Aînés', en lingala) et responsable de l'association anversoise Mwinda Kitoko ('Belle lumière', en lingala). Une immersion de plus de dix ans dans le milieu de la jeunesse qui a permis le suivi, d'une part, de quelques centaines de jeunes belgo-congolais ou d'origine africaine subsaharienne inscrits dans des situations de ruptures sociales, scolaires et familiales et, d'autre part, de partenariats, négociations ou conflits 
entre associations congolaises et pouvoirs communaux des villes de Bruxelles et d'Anvers.

Le troisième co-auteur, chercheur spécialisé dans le domaine des migrations de populations musulmanes, a séparément interviewé ses co-auteurs à trois reprises. Au cours de ces situations de co-analyse, l'hypothèse d'un lien entre les survivances coloniales dans les politiques sociales et les problèmes sociaux de la jeunesse issue de l'immigration originaire du Congo s'est déclinée autour de trois thèmes. Premièrement, les spécificités de l'immigration congolaise en Belgique et la situation sociale particulière des familles qui en découle. Deuxièmement, les spécificités de la condition juvénile des Belgo-Congolais, dont la thématique des dites bandes urbaines apparaît comme emblématique. Troisièmement, les spécificités du monde associatif congolais en Belgique, et ses relations avec les pouvoirs communaux d'Anvers et de Bruxelles en matière de politiques de jeunesse. Les textes issus de ces entretiens ont été commentés et retravaillés deux fois consécutivement par l'ensemble des auteurs afin de fournir le matériel de base du présent article.

Après avoir restitué dans une première partie les logiques d'émergence de quartiers africains à Bruxelles et à Anvers concomitamment de la sédentarisation des Congolais en Belgique, nous nous intéresserons dans la seconde partie aux dynamiques identitaires des jeunes que l'on peut qualifier de double absence identitaire au regard des conditions de migration et d'insertion de cette population dans l'ancienne métropole. Dans la troisième partie, nous nous pencherons sur le phénomène des «bandes urbaines » en ce qu'il exacerbe les ruptures sociales précédemment observées et cristallise un malaise postcolonial. Enfin, nous examinerons dans la quatrième partie, les liens entre les pouvoirs publics ayant en charge les politiques locales de la jeunesse et le milieu associatif belgo-congolais afin de dégager les particularités de ce groupe voire les particularités du traitement étatique à l'égard des Congolais. A la faveur d'un cadre comparatif, nous verrons que les situations postmigratoires marocaine et turque - migrations anciennes, de travail, non qualifiées et musulmanes - tendent à être mobilisées par les pouvoirs publics comme prototype généralisable.

\section{Emergence de quartiers « africains » à Bruxelles et à Anvers}

L'évolution de la présence congolaise en Belgique, du statut majoritairement étudiant à celui de demandeurs d'asile et de clandestins plus ou moins qualifiés (Mayoyo 1995 ; Kagné 2001 ; Martiniello et Kagné 2001) donne à voir à partir 
des années 1990, une diversification significative de ce groupe (Schoonvaere 2010) aujourd'hui constitué de trois générations.

L'avènement d'une «immigration congolaise » en Belgique est également concomitant d'une augmentation des demandes d'asile, qui conduit le gouvernement belge à répartir les demandeurs dans les différentes communes du pays, en vue de limiter la concentration d'étrangers à Bruxelles (Norro 1990). Des villes flamandes, à la tête desquelles Anvers - qui concentre aujourd'hui le plus grand nombre de Congolais établis en Flandre (Schoonvaere 2010: 63) se retrouvent confrontées à l'arrivée massive de demandeurs d'asile dans un contexte marqué par l'envol électoral du Vlaams Blok (devenu Vlaams Belang en 2004), le parti nationaliste flamand, durablement installé sur l'échiquier politique local (Boussetta 1998). Pour les acteurs de terrain, c'est la méfiance des propriétaires locaux, confrontant la population congolaise de manière immédiate à un problème de logement, qui amène la ville d'Anvers à proposer l'installation des Subsahariens, majoritairement congolais, dans des immeubles sociaux inoccupés de la rive gauche de l'Escaut (Linkeroever).

Si cette politique du logement offre une solution à court et moyen termes aux immigrants, elle se révèle problématique à plus long terme. Le surnombre des nouveaux arrivants, s'ajoutant à l'absence significative d'entretien et d'aménagement par la commune, génère une insalubrité des lieux et une stigmatisation de leurs occupants subsahariens, et congolais en particulier, durant la décennie 1990.

Les plus anciennement installés, bénéficiant d'une amélioration de leur situation financière ou de la régularisation de leur titre de séjour, finiront par quitter Linkeroever. Bien que correspondant à une amélioration des conditions de vie, ces départs constitueront un déracinement pour de nombreux jeunes. Pour maintenir leurs liens amicaux, certains prendront l'habitude de revenir à Linkeroever ou de se retrouver en d'autres endroits de la ville.

Ce mode d'investissement urbain, que nous avons également observé à Bruxelles, contraste avec celui des jeunes d'origine marocaine et turque, significativement attachés à leur quartier. L'immigration ayant été simultanée dans le cas de ces derniers, des réseaux familiaux ont pu se consolider en phase avec des logiques résidentielles concentrées sur certains quartiers (Manço 2012a: 29-34). La plus grande mobilité (et par là, visibilité) des Belgo-Congolais dans l'espace urbain tant anversois que bruxellois traduit un investissement de l'espace tout à fait inédit. Il est, au passage, important de relever que des tensions ont pu naître localement entre ces jeunes, devenus des «nomades urbains » à la recherche de leur semblables dispersés dans la ville, et les jeunes Belgomarocains que l'on peut considérer comme des « sédentaires urbains » qui ne 
s'aventurent guère hors de leur quartier. A différentes reprises, ces derniers ont eu l'impression d'être envahis sur « leur» territoire.

D'autre part, la discrimination à l'accès au logement, signalée dans le cas anversois, est loin d'être absente dans la région de Bruxelles-Capitale, et constitue un facteur de canalisation explicite vers les quartiers défavorisés (Lusanda 1993; Mazzocchetti 2011). A Bruxelles (constitué de 19 communes), hormis certains quartiers populaires dans les communes d'Anderlecht, de Bruxellesville ou de Molenbeek, c'est la commune de Saint-Josse qui constitue l'espace d'habitation majeur des Congolais (Schoonvaere 2010), à l'instar du quartier Linkeroever à Anvers, tandis que le célèbre quartier congolais du nom de Matonge, dans la commune voisine d'Ixelles, est avant tout un espace de commerce « exotique » et de loisirs où des Belgo-Congolais n'habitent qu'en petit nombre (de Clercq 2002 ; Corijn 2004 ; Demart 2013b).

Matonge n'a pourtant pas toujours eu la connotation négative qu'on lui prête aujourd'hui dans l'opinion publique et les médias. Jusque dans les années 1980, des « Belges du Congo » y venaient, par exemple, pour retrouver l'ambiance de Kinshasa. C'est à la suite du meurtre d'un jeune au début des années 1990 que des rumeurs ont commencé à se propager. D'après des commerçants riverains, certains médias bruxellois auraient contribué à la construction de l'équation « quartier africain = quartier chaud = insécurité » (Manço 2012b). Ces stigmates redevables de l'ethnicité n'épuisent toutefois pas l'émergence de formation identitaire hybride au sein de ce quartier traduisant la pluralité de référentiels, identitaires et spatiaux, de la diaspora (Swyngedouw et Swyngedouw 2009).

Dans un contexte tout à fait différent de celui des « quartiers » et banlieues françaises ou des ghettos américains, on peut se demander dans quelle mesure, ici aussi, les stigmates sociaux ne prennent pas leur source dans une insalubrité tributaire de la gouvernance locale et régionale. En l'occurrence, comment expliquer que les bâtiments soient décrépis, les espaces verts absents, de même que les terrains de jeu ou les bancs publics, alors que la même commune d'Ixelles abrite des quartiers dont certains sont cossus et fréquentés?

Les quartiers «africains » bruxellois et anversois (notamment l'équivalent anversois de Matonge, De Coninckplein) présentent de nombreuses similitudes: déclassement, insalubrité et insécurité ou, en tout cas, sentiment d'insécurité. Tous deux sont délaissés par les autorités et accusent un état de délabrement généralement avancé. Tous deux sont objet d'une couverture médiatique au ton parfois alarmant et sont fréquentés par des Congolais de différents milieux. C'est aussi dans ces quartiers que, depuis le milieu des années 1980, se sont déroulées de nombreuses manifestations ayant pour objet la politique congolaise (voir la contribution de Bodeux et Demart dans ce 
numéro). C'est enfin là que les Belgo-Congolais se sont confrontés à des violences policières et à des contrôles d'identité intempestifs et humiliants jusqu'au début des années 2000. A Bruxelles, cette agressivité policière contribuera à la formation de groupes de jeunes désireux de «se défendre » contre ces violences étatiques. D'un point de vue transrégional, on peut dire que la population belgo-congolaise de la commune de Saint-Josse et le quartier Matonge constituent un pôle d'attraction pour les Belgo-Congolais d'Anvers. Un nomadisme transurbain significatif qui connecte ces jeunes par-delà la distance géographique et les frontières linguistiques et administratives du pays. Mais avant d'entrer dans le détail de la question des dynamiques identitaires et des politiques locales, quelques éléments de contextualisation démographique s'imposent.

L'âge moyen de la population d'origine congolaise en Belgique était, en 2006, de 32 ans contre 41 ans pour la population belge de naissance (Schoonvaere 2010: 48). La structure des ménages dont le chef est né congolais est plus fragile d'un point de vue socio-économique : pauvreté, logement précaire, déménagements fréquents, etc. (Schoonvaere 2010: 66). Plus que pour toute autre population en Belgique ${ }^{1}$, cette structure est formée de ménages à personne unique $(37 \%)$ et de familles nombreuses de 3 enfants et plus (17\%). Pas moins de $25 \%$ de ces ménages sont monoparentaux (Schoonvaere 2010: 67). Il s'agit du taux de monoparentalité le plus élevé du pays. Néanmoins, les Congolais adultes en Belgique sont souvent scolarisés: $40 \%$ des hommes et $30 \%$ des femmes nés congolais ont terminé un cycle d'études supérieures contre $20 \%$ pour la population majoritaire, née belge, des deux sexes (Schoonvaere 2010: 70-71). Leur niveau de qualification contraste avec le niveau tout aussi élevé de chômage qu'ils connaissent. Contre 12 \% de chômeurs dans la population active totale de Belgique en 2010, $35 \%$ des Belgo-Maghrébins, $39 \%$ des Belgo-Turcs et $40 \%$ des Belgo-Congolais étaient des demandeurs d'emploi. Ce paradoxe pose la question de la non-reconnaissance des diplômes universitaires non européens, également de la discrimination à l'embauche (Tshibola, Mabaya et Makobo 2008: 81-84; Schoonvaere 2010: 72). En effet, un nombre significatif de diplômes a été obtenu en Belgique ou en Europe, parfois sur le mode du cumul des diplômes pour garder les «papiers » ou éviter un travail déqualifiant, ou encore le chômage (Mayoyo 1995). Mais surtout, pour ce qui nous intéresse ici, la situation qui en découle pour des personnes hautement qualifiées nuit à l'image que les jeunes ont de leurs parents, voire des études, et engendre de fortes tensions familiales.

\footnotetext{
1) Ces chiffres ne prennent pas en compte les personnes en situation irrégulière.
} 


\section{La formation identitaire des jeunes ou la « double absence » belgo-congolaise}

Les dynamiques identitaires que nous allons aborder à présent ont été observées et écoutées auprès d'environ 500 jeunes ayant eu affaire à des structures associatives dans le cadre d'un suivi scolaire, d'une médiation (famille, école, justice) ou d'un accompagnement socio-éducatif approfondi. Les données ont été recueillies au cours d'entretiens souvent répétés (liés au travail social), de discussions dans le cadre d'activités de groupe ou d'observation participante. Ce sont donc ici des grandes tendances qui sont relevées, permises par l'approche qualitative, et non les résultats d'une enquête quantitative. Sur la question identitaire proprement dite, une dizaine d'entretiens a été enregistrée avec l'accord des jeunes ${ }^{2}$.

Deux grandes catégories de jeunes peuvent être dégagées: ceux qui sont nés ou qui ont été socialisés en Belgique avant d'avoir atteint l'âge de la puberté, et ceux qui sont arrivés du Congo et d'autres pays d'Afrique à l'âge de 12-13 ans. De façon générale, les enfants et les adolescents congolais socialisés en Belgique connaissent peu leur pays d'origine. Pendant longtemps, ils n'ont pu s'y rendre du fait du coût élevé du voyage ou de leur statut juridique en Belgique. Cette situation tend à évoluer depuis 2003-2004. Une vague de régularisation du statut de séjour a touché les Congolais en 2000 et permis à certains d'envisager des séjours « au pays ». Toutefois, la majorité des jeunes nés en Belgique, rencontrés lors des activités supervisées par les deux co-auteurs travailleurs sociaux, n'ont pu voir leur pays d'origine (ni entretenir des liens avec leur famille élargie) avant l'âge de 15 à 20 ans $^{3}$. Ici aussi, on est loin de la situation des jeunes Belgo-Turcs et Belgo-Marocains, qui vont régulièrement dans le pays d'origine de leurs parents ${ }^{4}$. La «rupture» avec le pays d'origine est par ailleurs alimentée par ce qui apparaît comme un moindre accès de ces jeunes à l'univers médiatique du pays d'origine, au contraire de leurs parents et des jeunes des Belgo-Marocains ou Belgo-Turcs.

Outre la déconnexion territoriale, ce sont les ruptures symboliques, et plus précisément ce qui ne se transmet pas dans la parenté, ou d'une manière qui ne

\footnotetext{
2) Ces témoignages ont été recueillis par Mireille-Tsheusi Robert auprès des participants d'activités socio-culturelles que l'association $\mathrm{Ba} \mathrm{YaYa}$ a organisées à Bruxelles entre 2007 et 2012.

3) Beauchemin (2012) souligne que les Congolais d'Europe retournent en moyenne tous les 11 ans au Congo.

4) Pour des données quantitatives sur les jeunes belgo-marocains et belgo-turcs, voir les études de la Fondation Roi Baudouin disponibles sur Internet: Belgo-Marocains, entre deux rives (2008) et Belgo-Turcs, un pont ou une brèche entre la Turquie et l'Union européenne? (2006).
} 
correspond pas aux attentes des jeunes, qui sont à souligner. Car il en résulte des processus de réinvention identitaire chez les jeunes lesquels, en outre, soulignent les silences, cette fois-ci de l'école sur l'histoire partagée entre le Congo et la Belgique est criant.

Personne ne nous dit qui on est, d'où on vient, c'est quoi être Noir dans ce pays. Personne ne parle, ni à la maison, ni à l'école. (Danielle, 17 ans, née en Belgique ${ }^{5}$ )

Je sais de la colonisation ce que mon prof d'histoire m'a dit! D'après ce que j'ai vu, c'était lié au commerce triangulaire ${ }^{6}$, c'est-à-dire qu'il y avait des Blancs qui allaient en Afrique pour aller chercher des Noirs à échanger contre de la marchandise et pour en faire des esclaves et les emmener en Europe. (Jessica, 18 ans, née en Belgique)

La pluralité des référentiels culturels des diasporas noires a pu être appréhendée sous l'angle d'une « double conscience », celle de l' « Euro-Black identity » (Gilroy 1993), un concept que l'on peut relier au concept militant de « double expertise » (Lazalo Ndoma et Robert 2009), mis en avant à Bruxelles pour penser un cadre socio-éducatif débouchant sur une «véritable» intégration des jeunes d'origine congolaise. Ce concept part de l'observation de l'identité dévalorisée des personnes africaines ou d'origine africaine, et de stigmates qui tendent à enfermer les jeunes dans une altérité. Eux-mêmes peuvent anticiper cette altérité, en particulier lorsque leurs horizons professionnels fusionnent avec les espaces de « réussite» que la majorité blanche attribue aux Noirs: le sport, la musique ou le show (dans le sens du spectacle, mais aussi de l'extraversion dans la vie quotidienne). Ces orientations ne vont pas sans susciter des conflits intergénérationnels, lorsque pour les parents et les adultes de la « communauté ${ }^{7}$ en général, les jeunes générations de Belgo-Congolais sont dites «perturbées », «perdues » ou à l'africanité « altérée ». De leurs parents, beaucoup de jeunes diront en revanche qu'ils sont trop traditionnels et pas assez intégrés à la vie en Europe.

Ces stratégies anticipatives, parmi lesquelles la déviance que nous allons aborder, évoquent une «double absence» (Sayad 1999) socioculturelle en ce qu'elles témoignent de l'absence d'existence socialement valorisante ici et làbas. Les jeunes font d'ailleurs fréquemment le lien entre la dévalorisation du/ des pays d'origine et des cultures afférentes - à travers les débats autour de l'immigration, les médias, etc. - et les expériences d'exclusion sociale vécues en Belgique, à commencer par celles de leurs parents auxquels ils reprochent

\footnotetext{
5) Prénom d'emprunt, de même que pour les témoignages suivants.

6) Le commerce triangulaire relève de l'histoire de l'esclavage et non de la colonisation.

7) Pour reprendre le terme utilisé dans les milieux congolais.
} 
d'avoir courbé l'échine. Dans cette perspective transgénérationnelle, la discrimination n'est pas seulement pour eux une évidence, mais un « destin » face à la norme de la société.

Si l'identité, en l'occurrence culturelle, ne peut être décidée ni fixée une fois pour toutes (Hall 1992), l'altérité produite ou suggérée par les référents dominants peut, en revanche, faire l'objet d'une intériorisation (Swyngedouw et Swyngedouw 2009) qui n'exclut pas la référence à une identité panafricaine supposée embrasser les temps et les espaces (Segal 1998).

Dans leur recherche du Congo et de l'Afrique subsaharienne en général, soulignent les responsables et militants associatifs, c'est souvent de manière anachronique et confuse que les jeunes s'approprient les victoires africaines contre les envahisseurs européens, les idéaux panafricanistes, le concept de la négritude ou encore le mouvement Rastafari. Si l'intérêt pour l'Afrique passée, victorieuse ou sa diaspora renaissante est fort et intelligible à travers l'intensité des circulations d'information sur les réseaux sociaux, il n'empêche l'expression en parallèle d'un discours sur le rejet de l'Afrique actuelle, voire de leur propre africanité. L'auto-dépréciation se donne à entendre, ainsi que l'envie de «troquer » une identité d'Euro-Africain emprunte de stigmates contre une image idéalisée ou valorisée par la société globale, d'Africain et le plus souvent de «Black». L'orientation vers l'univers afro-américain, plus valorisé dans la société belge, apparaît comme ailleurs (Prévos 2003; Tshimanga et al. 2009) comme une des alternatives identitaires, d'autant que la réussite scolaire ne suffit pas toujours à se sentir accepté par la majorité.

Le jeune Noir est dans son monde, moi je parle d'expérience (...). Ce que je trouve triste c'est que le Noir ne voit pas plus loin que le bout de son nez, il ne veut pas découvrir les choses. Les Blancs, les Arabes, les Chinois, même s'ils ont une part de tradition, ils aiment découvrir les choses tandis que nous, on est des gros p-a-r-e-s-s-eux! Tout ce qu'on sait faire c'est écouter les punda (i.e. tam tam), les Werra (i.e. Werrasson, chanteur congolais), les kisanola (i.e. danse populaire), pointer (poignarder) les gens et parler comme ça : «âazi ». (Thierry, 20 ans, né en Belgique).

Il n'est pas rare que le terme péjoratif «âazi » (nègre en arabe), utilisé par les jeunes d'origine marocaine pour désigner les jeunes d'origine congolaise et africaine subsaharienne, soit repris par ces derniers pour s'appeler entre pairs. Outre qu'il n'y a pas d'équivalent «racial» avec le terme lingala «Bandibu » (nom d'une tribu au Congo, réputée belliqueuse) par lequel les Congolais désignent les «Arabes », le terme Bandibu ne traverse pas la frontière ethnolinguistique: il n'est pas connu des jeunes d'origine marocaine et n'affecte donc pas les sujets de la désignation. 
Sans entrer dans le détail de ces rapports inter-communautaires, on peut dire qu'à l'instar de l'appellation américaine « nigger », on a affaire à une appropriation du stigmate qui fait identité, et qu'il est intéressant de mettre en perspectives avec une forme de violence a priori universelle (le phénomène des bandes) mais dont la déclinaison locale traduit des processus de racialisation propres au contexte belge.

\section{Les «bandes urbaines », une particularité de la jeunesse congolaise?}

Le phénomène des «bandes urbaines », hautement médiatisé en Belgique ${ }^{8}$, tend à exacerber des dynamiques de rupture sociale que l'on observe de manière globale chez les jeunes d'origine congolaise et par extension d'origine subsaharienne. Forme avancée de marginalité, ces groupes expriment la difficulté de leurs membres à sortir de carcans identitaires ethno-raciaux (Mohammed 2011) produits par la société globale (Fassin et Fassin 2006 ; Solomos 2003). En 2009, environ 25 bandes urbaines étaient répertoriées par la police bruxelloise, dont les membres (ayant majoritairement de 15 à 19 ans) sont tous d'origine africaine subsaharienne et porteraient des armes blanches (couteaux, scies pliables, voire machettes). Depuis 2002, l'association $\mathrm{Ba} Y a Y a$ a recensé environ 2500 jeunes impliqués de près ou loin dans ces formations urbaines. Le noyau de ces regroupements (18 sur 25) est toujours congolais. Une douzaine de morts (une trentaine selon les jeunes que nous avons rencontrés), liée à des bagarres entre groupes rivaux, est à déplorer à Bruxelles depuis 2002, dont 6 depuis la fin de 2009 .

La police locale ${ }^{9}$ attribue à ces groupes des meurtres, des coups et blessures, des vols qualifiés et du trafic de stupéfiants. A l'encontre du point de vue policier faisant état du caractère très minoritaire de la pratique du viol au sein de ces groupes, le point de vue associatif estime le nombre de viols collectifs sur jeunes filles d'origine subsaharienne ou métisses en augmentation depuis plusieurs années. La sous-représentation statistique étant liée au fait que les victimes portent rarement plainte. De même que la présence congolaise en Flandre est plus tardive qu'à Bruxelles, la formation de «bandes urbaines » (stadsbendes) date de 2005. Elle s'est organisée autour de jeunes d'Anvers qui ont fait appel à leurs comparses bruxellois, avec lesquels ils avaient des liens familiaux, pour obtenir main-forte lors de bagarres avec des groupes rivaux.

8) La presse les a également nommés « gangs noirs » ou «bandes de Blacks ».

9) Cf. la circulaire du Parquet de Bruxelles «Stadsbendes-Bandes Urbaines », 28/12/1999. 
Bien que la violence associée à ces sociabilités soit incontestable, la notion de « bandes urbaines » nous semble à reconsidérer au regard des stigmates que cette appellation draine (Mohammed et Muchielli 2007). En outre, ces groupes ne se constituent pas autour d'un objet illégal et/ou pécunier, mais sur base résidentielle et d'expériences communes. Les activités de ces groupes sont d'abord sociales et artistiques, et non lucratives.

Si tous les jeunes issus de l'immigration congolaise, qu'ils soient nés en Belgique ou au Congo, ne versent pas dans la violence urbaine à la faveur d'autres espaces de socialisation ou d'expression (Jamoule et Mazzocchetti 2011), néanmoins les formes de cette violence interpellent, notamment en comparaison avec la situation des jeunes Belgo-Marocains et Belgo-Turcs. En l'absence d'analyses sociologique ou psychologique explicatives des causes du phénomène, l'expertise associative et militante (Lazalo Ndoma et Robert op.cit.) appelle à considérer deux hypothèses. La première est la socialisation déstructurée de jeunes, du fait des ruptures dans la parenté qu'engage la migration. La seconde est leur autodépréciation, tributaire d'assignations statutaires à caractère racial et colonial.

La déstructuration du tissu et de l'économie familiale que génère le processus migratoire est-elle en cause ? Outre les situations de monoparentalité déjà relevées, qui contrastent avec la majorité des familles belgo-marocaines et belgo-turques, ce sont les conditions migratoires des Congolais qui participent de l'éclatement de la parentèle, car elles ne permettent pas l'arrivée simultanée des membres d'une même famille nucléaire sur le territoire du pays d'accueil.

Dans certains cas, les familles sont dispersées sur plusieurs pays européens, parfois pendant plusieurs années durant lesquelles les enfants peuvent être livrés à eux-mêmes, y compris lorsqu'ils sont chez une tante ou un oncle. Inversement, l'éloignement d'avec les oncles ou les tantes n'est pas sans conséquence en termes d'éducation familiale. Outre l'autorité partagée, ce sont les fonctions des différents membres de la parenté dans le processus de socialisation et d'éducation qui se trouvent amputées. Par exemple, face à l'absence d'une tante supposée faire l'éducation sexuelle de sa fille (sa nièce), la mère biologique ne sera pas nécessairement outillée ou légitime pour prendre le relais d'une fonction qui ne lui aura pas été transmise. La socialisation incomplète de nombre de jeunes de cette origine peut dès lors apparaître comme un facteur de ruptures sociales.

L'autre hypothèse avancée par certains experts associatifs et militants, celle du manque d' « estime de soi » ou de la « souffrance identitaire » des jeunes renvoyant au rejet de leur être, discriminé et invisibilisé -, a pour partie liée avec le traitement mémoriel de l'histoire coloniale belge qui, tel un tabou, ne 
leur est contée ni dans le milieu scolaire ni dans le milieu familial. L'absence de transmission scolaire et parentale sur la colonisation, sur l'histoire partagée entre société d'appartenance/résidence et d'origine, apparaît comme le catalyseur de bon nombre de frustrations et d'extrapolations. A ce stade, on peut se demander dans quelle mesure les jeunes belgo-congolais socialisés en Belgique ont intériorisé les préjugés et stéréotypes raciaux véhiculés par la société belge via les médias, la publicité et le folklore, sur l'infériorité des Noirs en général et des Congolais en particulier.

La relation que les jeunes belgo-congolais vivent par rapport à la société blanche majoritaire est empreinte d'un sentiment de domination, qu'ils relient à l'esclavage et à la colonisation au regard de l'exclusion ressentie et de la discrimination vécue. Certains tendent d'ailleurs à considérer que leurs aînés, dont leurs propres parents, ont fait preuve de docilité envers la « domination blanche »; une perception qui leur interdit dans bon nombre de cas de les prendre en modèle. Leur reproche vise aussi le silence relatif à l'histoire coloniale et migratoire ; une histoire pouvant évoquer humiliations et douleurs chez les parents qui reconnaissent préférer ne pas en parler, même si des initiatives associatives allant dans le sens des demandes juvéniles se mettent en place.

La thèse de l' «automutilation» a été développée par certains acteurs associatifs pour défendre l'idée selon laquelle en s'attaquant à son semblable (Lazalo Ndoma et Robert 2009), le jeune Noir socialisé en Belgique, en manque de reconnaissance sociale et en mal d'estime de soi (Lagrange 2000), se poignarde en poignardant son alter ego. Dans cette perspective, le jeune n'aime pas ce qu'il pense être : l'image que lui renvoie le regard des Blancs. Il n'aime pas ce que ses parents sont devenus. Il n'aime pas la manière dont son pays de naissance (la Belgique pour la plupart d'entre eux) l'accueille et le considère. La dépréciation générale des Noirs africains, le sentiment d'infériorité et la tendance au repli auprès des pairs que cela induit, mais également les notions d'honneur et de « garder la face » présidant aux querelle et bagarres : voilà quelles seraient les causes d'une dynamique auto-destructrice dont les déclencheurs sont souvent futiles (Esterle 2007) : un mauvais regard, la traversée non autorisée d'un territoire, etc.

Ces hypothèses, qui tendent à désigner le contexte social et historique comme étant à l'origine de cette forme singulière de violence entre Noirs, peuvent être problématiques pour les responsables municipaux du pays d'accueil, en particulier parce qu'elles posent la question du cadre mémoriel permettant à des jeunes de s'identifier, en tant que Belges d'origine congolaise, aux grands récits nationaux. Pour les associations militantes, l'insuffisante prise en considération par les pouvoirs municipaux de ces faits de violence semble liée au 
fait que celle-ci se déroule entre Congolais ou entre Noirs. L'idée selon laquelle les Africains ont un caractère «bon enfant» leur semble encore prégnante. Or c'est ce genre de présupposé qui a amené à relativiser les problèmes de cette jeunesse (Lazalo Ndoma 2006) dès les années 1990, au contraire de celle de la communauté belgo-marocaine qui, elle, aurait de « sérieux problèmes ».

Pour les pouvoirs institutionnels, les demandes de reconnaissance des associations mettant en avant la nécessité de penser d'autres types de suivis socio-éducatifs relèvent souvent du communautarisme. Dès lors, la mise en œuvre d'une politique de la jeunesse censée répondre aux besoins spécifiques des jeunes Belges d'origine congolaise est un point de désaccord majeur entre les pouvoirs municipaux de Bruxelles et d'Anvers et les associations belgo-congolaises.

\section{Les politiques locales de jeunesse et le milieu associatif belgo-congolais}

L'absence relative de données spécifiques à propos des jeunes issus de l'immigration congolaise et plus généralement subsaharienne - en termes d'échec et de décrochage scolaires ${ }^{10}$, par exemple - pourrait expliquer la tendance des pouvoirs publics à se référer aux modes d'encadrement et aux pratiques socioéducatives pensés pour les jeunes issus des migrations maghrébine et turque (Cartuyvels et Hebberecht 2001; Rea 2006). Ces politiques locales d'encadrement préventives sont à tendances occupationnelles et surtout sécuritaires, en vue d'éviter d'éventuels troubles urbains (Manço 2012a: 66-69), malgré les efforts de démarcation du monde associatif professionnel en faveur d'un travail éducatif. Outre que cette politique n'apparaissent pas appropriées aux associations belgo-congolaises, il semble que les jeunes belgo-congolais n'adhèrent pas à ces dispositifs, préférant improviser des activités en extérieur : dans la rue, aux alentours des maisons de jeunes, dans les parcs, dans les « quartiers africains », etc. Cette situation qui perdure depuis la fin des années 1980 à Bruxelles, et depuis 2000 à Anvers, a amené le milieu de la diaspora congolaise à se constituer en associations pour porter des revendications.

10) Outre la recherche détaillée déjà citée de Jamoule et Mazzocchetti et les données de l'étude Foyer (2010a ; 2010b) établissant une différence de performances scolaires entre jeunes d'origine rwandaise et congolaise, on peut citer les travaux de Jacobs (2009), Jacobs et Rea (2011). 
Dès la fin des années 1980, des associations à but socio-éducatif sont donc créées par des individus ${ }^{11}$, à la suite des Eglises (Demart 2008), en vue de pallier les carences institutionnelles auxquelles se confrontent les Congolais (Cornet 2004). Après 30 années d'activités dans le cas francophone et 20 années de présence dans le cas néerlandophone, ce milieu associatif demeure toutefois peu connu des pouvoirs publics - notamment au niveau municipal -, qui le considèrent comme désorganisé et anarchique (Gatugu et al., 2004 ; Kagné 2008). Il en résulte une certaine incohérence dans les relations entre ces associations et les autorités francophones ou flamandes ${ }^{12}$.

Dans un premier temps, les responsables communaux, tant bruxellois qu'anversois, estimèrent que les associations issues des milieux congolais n'étaient pas suffisamment représentatives, et posèrent comme condition à une reconnaissance et à l'octroi de subsides, la fédération des différentes structures portées par des personnes d'origine subsaharienne. Or, cette injonction, qui n'est pas exigée des autres populations issues de l'immigration, donne à voir un traitement hautement problématique, puisqu'en balayant toute la diversité nationale, culturelle, linguistique et religieuse de la catégorie «Afrique subsaharienne » - rien qu'à Bruxelles, cette catégorie englobe plus de 45 nationalités - l'Etat racialise ce secteur associatif.

Dans le cas anversois comme dans le cas bruxellois, l'exigence de fédération est apparue comme un « piège » pour le monde associatif congolais et plus généralement subsaharien, au vu du temps et de l'énergie requis au détriment du travail de terrain et de la gestion des problèmes socio-éducatifs. Sans reprendre à notre compte la notion de piège, on peut s'interroger sur le fondement de ces exigences. Car même si la catégorie plurielle de «subsaharien » se réfère à une population de plus petite taille que celle de «marocain » (Schoonvaere et Schoumaker op.cit.), elle n'en demeure pas moins paralysante sur le long terme pour l'accès aux possibilités de financement de ce secteur associatif.

11) Par exemple, Lisanga ya ba mama zaïroise na Belgique (1985), Fungola (1987), devenu BaYaYa en 2002 ou La zaïroise et ses sœurs (1990), Le carrefour des jeunes Africains (1996) du côté francophone. Du côté néerlandophone, les premières associations voient le jour à partir de 1990, comme Mwinda Kitoko à Anvers.

12) Certaines réunions publiques ont été organisées par les associations subsahariennes anversoises, parmi lesquelles l'association Mwinda Kitoko, et des échevins furent invités. Les échevins (conseillers municipaux) anversois étaient impressionnés de ce qu'ils avaient entendu de la part des responsables associatifs, congolais et autres qui y ont pris la parole afin d'exposer leurs problèmes. Les politiciens locaux n'ont rien trouvé de mieux à dire qu'ils ne savaient pas que des Subsahariens d'Anvers pouvaient parler aussi bien le néerlandais ! 
Ainsi, dans les années 1990 à Bruxelles, une intense mais infructueuse période de fédération des associations fut entamée. Entre 2004 et 2005, pas moins de 11 tentatives de création de fédérations échouent. Parallèlement, de nouvelles stratégies naissent sans apporter nécessairement les résultats escomptés : les responsables associatifs bruxellois se rendent compte de l'opportunité de se présenter comme «subsaharien » plutôt que « congolais » dans leurs dossiers de demandes de subsides adressés aux communes, mais sans que cela n'engage forcément une réelle dynamique collective. L'initiateur de l'une de ces fédérations associatives avortées se souvient :

Tous les Congolais veulent être appelés « mopréso » («mon président ») et pouvoir au moins une fois dans leur vie, exercer ce qu'ils ont appris dans les universités belges. On vous dira que nous ne savons pas nous organiser, mais toute une vie sans travailler, c'est dur quand on a des compétences. Alors on crée quelque chose où l'investissement de départ n'est pas vraiment pécunier : un groupe sportif, une église ou une association. Mais vraiment, cette situation était ingérable, car quand quelqu'un acceptait d'être secrétaire général adjoint, il finissait toujours par entrer en conflit avec le numéro un. Mais comme ils provenaient de différentes ASBL ${ }^{13}$, ça finissait toujours par la scission des alliances ou des plateformes. (Associatif belgo-congolais, en Belgique depuis 1999 ; interview, 2012).

À Anvers, le travail de fédération des associations subsahariennes, toutes nationalités d'origine confondues, a duré 7 ans, de 1993 à 2000. Il a impliqué des associations aussi différentes que les origines de leurs fondateurs, leurs histoires, leurs idées porteuses et leur mode de fonctionnement. Outre la crainte des associations de perdre leur autonomie et spécificités en devenant membre d'une fédération, frictions et concurrence sont régulièrement venues parasiter cette entreprise de fédération, rappelant que la «conscience africaine » n'est pas une donnée mais un construit, comme en témoigne le caractère marginal des tentatives panafricaines au sein de ces milieux associatifs (Grégoire 2010). À Anvers, dans un contexte encourageant le multiculturalisme, bien que de manière parfois contradictoire (Bousetta 1998), la dynamique aboutira. Les acteurs de l'époque soulignent que la persistance des problèmes sociaux et des discriminations (notamment, l'exposition des Subsahariens aux contrôles policiers intempestifs, l'accès au logement, à la scolarité, etc.) a contribué à l'émergence d'une plateforme de revendications communes à toutes les associations subsahariennes existantes. Créée en 1999, cette fédération est financée par les pouvoirs publics flamands depuis 2001. La Plateforme panafricaine est aujourd'hui porte-parole des Subsahariens à Anvers et regroupe 22 nationalités, même si le groupe prépondérant reste congolais.

13) Association Sans But Lucratif. 
Cependant, en matière d'aide à la jeunesse, la fédération panafricaine d'Anvers s'est d'abord retrouvée impliquée dans un système préétabli : l'administration communale finançait déjà un certain nombre d'associations belges ou marocaines chargées d'encadrer les jeunes issus de l'immigration. La fédération panafricaine fut invitée à collaborer avec elles afin d'obtenir des subsides, alors même que ces dernières entendaient préserver leurs acquis. Outre qu'elles ne partagèrent guère leurs subsides avec les nouveaux venus subsahariens, leur offre de services et d'activités ne répondait pas aux attentes de ce public. Dans un contexte où le fonctionnement général et l'organisation des activités ne donnait pas droit de cité à la plateforme africaine, les militants subsahariens eurent le sentiment explicite d'être perçus comme une concurrence pour les structures existantes.

À ceci s'ajoute l'asymétrie des statuts puisque les associations congolaises étaient composées de bénévoles formés tandis que les associations belges et marocaines, professionnelles et agréées, employaient des cadres formés et salariés, qui bénéficiaient d'une sécurité de l'emploi. S'est alors imposé le sentiment d'être mis sous tutelle par les pouvoirs subsidiants et d'être contraint de collaborer avec des associations professionnelles prenant l'ascendant sur les bénévoles d'origine congolaise, non pas en raison de compétences sur le terrain ou de diplômes mais de légitimité auprès des pouvoirs publics. La plateforme africaine d'Anvers bénéficie aujourd'hui de financements structurels, mais ceux-ci sont sans commune mesure avec ceux d'autres fédérations autochtones. Il en résulte des tensions liées au fait que les procédures d'accès aux financements ne tiennent pas compte des spécificités de ce secteur associatif et d'un public ciblé qui continue d'être méconnu des institutions.

Malgré un contexte et des politiques d'intégration différenciées (Adam 2011), le constat d'une subsidiation marginale et soumise à des tutelles est le même à Bruxelles où les structures associatives d'origine subsaharienne n'ont pas de financements structurels ${ }^{14}$. Les partenariats entre associations, parfois constitués à la demande des municipalités en vue de rétablir le lien social avec les jeunes (et/ou leurs parents), ont donné lieu à une série de situations où des associations congolaises/africaines, composées de bénévoles, se sont vues collaborer avec des structures belges, professionnelles, qui énonçaient les objectifs et rythmes de travail afin de bénéficier de subsides ponctuels.

Il en résulte alors, de façon systématique, que telle association « belge » reçoit les subsides publics au lieu de l'association « congolaise » ou « subsaharienne »

14) Ceci suppose une subsidiation récurrente couvrant les frais de fonctionnement, de location, de personnel et d'activités. 
qui les aura demandés au départ, et à qui cela aura été accordé « sous condition ». L'association congolaise effectuera le travail d'animation sociale ou d'encadrement éducatif sur le terrain, comme convenu avec le pouvoir communal, mais sous la supervision de l'association «professionnelle » sollicitée par ce même pouvoir. L'association «professionnelle» gèrera le budget du projet et remboursera les dépenses de l'association active sur le terrain sur présentation de preuves de paiement, lorsque cela lui semble justifié. Sans nous attarder sur les tensions que cela a pu engager, on ne peut manquer de relever le paradoxe de ces pratiques institutionnelles de mise sous tutelle. Car si le manque de confiance des pouvoirs publics envers les représentants du monde associatif subsaharien est patent, il n'en va pas de même à l'égard du travail qu'ils effectuent. Dans la pratique, ce fonctionnement est problématique puisque l'ordre institutionnel ne rencontre pas nécessairement les besoins du terrain, à l'exemple des activités ayant lieu la nuit entre jeunes dans les parcs et pouvant aboutir à des affrontements et décès (cf. supra). La logique institutionnelle (horaire de bureaux, subsides distribués au compte-gouttes, soupçon de mauvaise gestion inhérente aux Africains, etc.) crée alors une atmosphère « paternaliste » qui affecte d'autant plus les militants que cette asymétrie hiérarchique a lieu dans le cadre de rapports ethniques racialisés.

En outre, pour accorder un financement, les autorités publiques tant bruxelloises qu'anversoises imposent souvent des objectifs ponctuels de type culturel et récréatif. Pour les associations, il s'agit là de meubler les loisirs et les périodes de vacances des jeunes Belgo-congolais qui relèguent au second plan toute action socio-éducative en profondeur et de longue haleine, pouvant comprendre des activités culturelles mais sans s'y réduire ${ }^{15}$. Les conflits qui en résultent sont d'autant plus violents que les associations se voient mobilisées pour le travail de terrain et leurs compétences reconnues sur base de critères objectifs (diminution de conflits entre bandes et de décès, raccrochage scolaire, etc.), mais leurs analyses disqualifiées au titre de leur communautarisme.

15) L'association $\mathrm{Ba} Y a Y a$ a notamment proposé qu'il soit question d'une « différenciation du mode d'investissement de l'espace urbain » car les jeunes belgo-congolais sont plus mobiles que, par exemple, leurs homologues belgo-marocains et ne se réunissent pas forcément dans leurs quartiers ou dans des locaux associatifs, qui deviendraient leurs lieux habituels. L'association propose également des pratiques socio-éducatives visant à susciter la revalorisation de l'identité africaine et la réhabilitation de l'histoire coloniale dans le patrimoine belge. Jusque là, ces activités étaient jugées à caractère « ethnique » ou « communautariste » et n'étaient pas validées par les autorités communales, mais le Ministère de la Jeunesse vient de financer une rechercheaction visant précisément à valider ce dispositif expérimental. 
Mais surtout, le fait que l'administration communale fasse appel, en guise de référent, à une association professionnelle reconnue et «belge ${ }^{16}$ - qui n'est cependant pas nécessairement impliquée dans le projet négocié (entre association belgo-congolaise et pouvoir subsidiant) ni ne maîtrise les spécificités des migrations congolaises ou subsahariennes (ou de la problématique: «bandes urbaines », «jeunes fréquentant Matonge», etc.) -, est vécu comme un paternalisme reproduisant l'asymétrie hiérarchisante du temps colonial.

C'est enfin la thématique récurrente du « renvoi à l'Afrique » (réelle et fantasmée) dans les interactions associées à ces partenariats qui est soulignée comme problématique par certains responsables associatifs. Lors de réunions de travail, par exemple, il n'est pas rare que des responsables emploient des expressions telles que : «Ce n'est pas le Congo, ici !» ou « Il ne faut pas se comporter comme dans une jungle» pour mettre un terme aux revendications militantes allant dans le sens de réformes des modes de fonctionnement professionnels et de la prise en compte du contexte postcolonial. Outre la vexation, lorsque les responsables associatifs font remarquer que ces expressions sont déplacées, c'est le lien personnel des acteurs institutionnels belges avec le Congo qui ressort. Selon les acteurs associatifs, il s'agirait ici souvent des responsables municipaux les plus âgés, nés avant l'indépendance du Congo. Bon nombre d'entre eux auraient des parents ayant vécu au Congo ou ont été eux-mêmes coopérants lors de périodes plus récentes. Pour les responsables associatifs, ceci explique leur tendance à « reconstituer une relation affective de subordination » significative d'un «paternalisme postcolonial» qui touche à notre sens à la question du « rapatriement de la colonie » en métropole (Lebovics 2004), et plus généralement, à la culture coloniale (Jacquemain, op.cit.; Viaene et al. op.cit.) et à ses modes de transmission (Licata et Klein op.cit.; Vanderlinden 2004) dans les institutions : un thème peu exploré au sein du monde académique.

\section{Conclusion}

Le renvoi à l'Afrique des acteurs associatifs durablement assignés à leur identité d'origine dans leurs interactions avec les pouvoirs publics belges contraste avec la double absence éprouvée par les jeunes, en même temps que ces désignations signalent en miroir ce qui peut relever des problématiques

16) Nous nous basons ici sur plus de 5 projets menés sur de longues durées (de plusieurs mois à quelques années) avec différents partenaires. 
identitaires autochtones vis-à-vis des descendants de leur ex-colonie et surtout de leur présence en Belgique. La permanence de frontières raciales que nous avons restituée à partir de différents angles s'articule à la configuration postcoloniale, non pas parce que la situation post-migratoire des Congolais serait paradigmatique, mais parce que ce groupe constitue le noyau organisateur des dynamiques associatives africaines de Belgique, d'une part, et d'autre part, parce que l'imaginaire belge vis-à-vis des Africains ou le rapport belge aux Noirs s'est, de par l'histoire coloniale, constitué dans le rapport, effectif ou virtuel, avec les Congolais.

L'émergence de quartiers africains en Flandre comme en région bruxelloise faisant l'objet d'une négligence de la part des pouvoirs locaux est un premier indicateur de la composante (post)coloniale de l'action publique, ou pour le moins de la hiérarchisation des priorités étatiques. Les données démographiques faisant état d'un taux de chômage record au sein d'un groupe pourtant détenteur d'un niveau de qualification qui le distingue de toutes autres populations d'origine étrangère, puisqu'il équivaut globalement à celui de l'autochtonie, sont en soi révélatrices des implicites racistes travaillant la société globale, à l'échelle des individus comme des institutions, privées ou publiques. Mais elles sont aussi l'expression d'un contexte au sein duquel des jeunes socialisés en Belgique doivent trouver leur place. Outre les silences de l'école sur l'histoire, reflétant un récit national faisant l'impasse sur les apports de la colonisation, et donc du Congo et de plusieurs générations de Congolais, ce sont les difficultés des parents à mettre en forme une histoire (coloniale et migratoire) dont ils sont en quelque sorte les grands perdants qui ressort du discours des jeunes comme le fondement de leur double absence, sociétale et culturelle, ou de dynamiques urbaines dont la violence est exclusivement exprimée entre soi. Une violence qui se chiffre en décès mais également en blessures graves, physiques et psychologiques.

Cette jeunesse s'avère être le point aveugle des politiques locales qui tendent à prendre comme modèle paradigmatique la jeunesse d'origine maghrébine. Les pouvoirs publics s'accordent pourtant à reconnaître que la jeunesse d'origine congolaise et africaine échappe à l'action institutionnelle, en particulier lorsqu'ils sollicitent les acteurs associatifs pour rétablir le lien social sur leur territoire. Paradoxalement, le mode de financement de cette action socioéducative s'oppose à des actions à long terme, structurelles lorsqu'il repose, et c'est souvent le cas, de manière exclusive sur le bénévolat des acteurs associatifs.

Une question fondamentale se pose alors pour ces militants: celle du lien entre la discrimination à l'embauche et la discrimination aux subsides pour les associations, en particulier celles qui évoluent à Bruxelles et en Wallonie, 
même si le changement de pouvoir à Anvers (avec l'élection de la N-VA, un parti nationaliste et xénophobe) laisse augurer un possible démantèlement de l'acquis de ces associations. Des recherches ultérieures doivent êtres menées pour mieux saisir les mécanismes de ces discriminations, du point de vue de l'autochtonie, mais aussi les stratégies mises en place par les acteurs pour les contourner. Le modèle multiculturaliste de la Flandre offre des ressources évidentes comparativement à la Wallonie et à Bruxelles, et peut expliquer le fait que de plus en plus d'associations congolaises sollicitent, à Bruxelles, des subsides flamands, après avoir fait le tour du fonctionnement francophone, tout en soulignant la brutalité du «racisme flamand». Il serait intéressant de mener des recherches comparatives dans le domaine scolaire, socio-professionnel pour voir si là aussi, des espaces de contournement des frontières raciales sont observables ou pas.

En attendant, la méconnaissance des Belgo-Congolais par les pouvoirs publics est un élément transversal et structurel qui interpelle, de même que la tendance à situer dans le domaine du culturel des problématiques sociales. Malgré un nombre significatif de rapports, de revendications et de recherches, les Belgo-Congolais se confrontent à Bruxelles comme à Anvers à leur éternel statut de groupe non identifié. Cette non-intégration dans le domaine de la connaissance apparaît à de nombreux Belgo-Congolais, y compris en dehors $\mathrm{du}$ monde associatif, comme l'expression implicite mais tenace d'un refus d'intégration.

Alors que les Marocains et les Turcs sont arrivés en Belgique bien après les Congolais, ils apparaissent bien plus (re)connus par les pouvoirs publics. Même si cela n'empêche les stigmates et les stéréotypes, force est de constater que ces derniers, certes plus nombreux, ont fait l'objet de bien plus d'attention sur le plan scientifique, politique et social (Martiniello et al. 2007), alors même que leur histoire partagée avec les Belges est récente (années 1960) comparativement aux Congolais (fin du 19e siècle).

« Les Congolais sont chrétiens, il ne doit pas y avoir trop de différence avec nous », déclarait un échevin (adjoint au maire) bruxellois, en 2007. On n'est pas loin du «Congolais bon-enfant» ni paradoxalement des «enfants-soldats », supposés constituer les «bandes urbaines », une représentation qui depuis les années 1990 empêche de penser ce phénomène comme un pur produit de la société belge. Ces représentations simplistes et fantasmagoriques de l'Autre, proche, témoignent de scories postcoloniales (Stoler 2008 ; Mbembe 2000) qui fragmentent le lien social et engendrent des tensions intercommunautaires au sein de cette société multiculturelle. Dès lors, une question se pose et gagnerait à faire l'objet de débats publics : doit-on voir dans le refus de connaître les 
Belges d'origine congolaise et plus généralement les personnes d'origine subsaharienne, une continuité avec la politique coloniale dont les logiques ségrégatives (Ndaywel 1998) refusèrent de penser l'intégration des indigènes dans l'espace social, économique et culturel de la métropole? À l'agenda de la recherche, il serait nécessaire d'éclairer d'une part, le no man's land dans lequel ce groupe se trouve et, d'autre part, le label racial exigé par les pouvoirs publics, lorsque ceux-ci conditionnent la reconnaissance et les subsides à l'avènement d'une structure « subsaharienne » (alors que la norme est celle de la reconnaissance des associations sur base de l'origine nationale) en l'absence de toute réflexion collective autour de la situation des Noirs de Belgique.

Enfin, les accusations récurrentes de communautarisme face aux analyses et revendications de ce secteur associatif se doivent d'être déconstruites en ce qu'elles entérinent bien souvent le constat d'une racialisation du financement associatif et se donnent à voir comme un argument facile pour disqualifier des acteurs en quête de reconnaissance et d'autonomie. Car, en définitive, et ce point gagnerait à être mis à l'ordre du jour de l'agenda de la recherche, c'est bien de l'anxiété postcoloniale à l'égard d'une définition post-raciale de l'identité belge qu'il s'agit. Dans cette perspective la compréhension de la marginalisation, économique, symbolique et juridique du secteur associatif belgo-congolais ou belgo-africain, par les politiques locales francophones et néerlandophones, en appelle à un décentrement du regard, vers l'autochtonie.

\section{Bibliographie}

Adam, I., Ben Mohammed, N., Kagné, B., Martiniello, M. et Rea, A. 2002. Histoires Sans-Papiers, Bruxelles : Editions Vista.

Adam, I. 2011. Une approche différenciée de la diversité. Les politiques d'intégration des personnes issues de l'immigration en Flandre, en Wallonie et à Bruxelles (1980-2006). In: Ringelheim, J. (éd.), Le droit et la diversité culturelle. Louvain-la-Neuve : Bruylant.

Bancel, N., Bernault, F., Blanchard, P. et Boubeker, A. 2010. Ruptures postcoloniales. Les nouveaux visages de la société française. Paris: La Découverte.

Beauchemin, C. 2012. Migration between Africa and Europe (MAFE). Paris: INED, http://www.ined .fr/fichier/t_recherche/NoteDetPhare2012/Po217.pdf.

Bousetta, H. 1998. Le Paradoxe anversois. Entre racisme politique et ouvertures multiculturelles. Revue européenne de migrations internationales, 14 (2): 151-172.

Cartuyvels, Y. et Hebberecht, P. 2001. La politique fédérale belge de sécurité et de prévention de la criminalité, Déviance et société, 25 (4): 403-426.

Coenen, M.-T. et Lewin, R. 1997. La Belgique et ses immigrés. Les politiques manquées. Bruxelles : De Boeck Université. 
Corijn, E. 2004. Matonge, centre multiculturel à Bruxelles. Politique, 35: http://politique.eu.org/ spip.php?articlen61.

Cornet, A. 2004. Les Congolais en Belgique au XIX et XXe siècles. In: A. Morelli (éd.) Histoire des étrangers ... et de l'immigration en Belgique de la préhistoire à nos jours. Bruxelles : Couleurs Livres. 375-400.

De Clercq, D. 2002. Everyday Urban Space in Matonge, OASE Journal for architecture: 62-85.

Demart, S. 2008. Le « combat pour l'intégration» des églises issues du Réveil congolais (RDC). Revue Européenne des Migrations Internationales, 24 (3): 147-165.

Demart, S. 2013a. Chronique d'une histoire orale à Matonge (Bruxelles). Revue Européenne des Migrations Internationales, à paraître.

— 2013b. Présence des Congolais à Bruxelles et postcolonialismes. Entretien. Agir par la culture, (33). Edition en ligne http://agirparlaculture.be/entretien/151-presence-des-congolaisa-bruxelles-et-postcolonialismes (dernier accès 29/03/2013).

- 2013c. Riots in Matonge and... the indifference of public authority?, Brussels Studies, Number 68, July ist 2013 .

Esterle-Hedibel, M. 2007. Controverses théoriques autour des bandes de jeunes. In: M. Mohammed et L. Mucchielli (eds.), Les bandes de jeunes, des 'Blousons noirs' à nos jours. Paris : La Découverte : 85-96.

Etambala, M. A. 1993. In het land van de Banoko: De geschiedenis van de Kongolese/Zaïrese aanwezigheid in België van 1885 tot heden. Leuven: Hoger Instituut voor de Arbeid.

Fassin, D. et Fassin, E. 2006. (dirs). De la question sociale à la question raciale : représenter la société française. Paris: La Découverte.

Foyer 2010a. De Congolese jongeren aan het woord. Een kijk op de schoolse en sociale situatie van Congolese jongeren in Brussel. Brussels: Foyer.

_ 2010b. Rwandese jongeren op Brusselse scholen. Het verhaal van de Rwandese jongeren en hun ouders over naar school gaan en leven in Brussel. Brussels: Foyer.

Gatugu, J., Amoranitis, S et Manço, A. (éds) 2004. La vie associative des migrants: quelles (re)connaissances? Réponses européennes et canadiennes. Paris : L'Harmattan.

Gillet, F. 2007, L'histoire coloniale en débat: examen d'une Belgique en repentir. Mouvements, 51: 70-77.

Gilroy, P. 1993. The Black Atlantic: Modernity and Double Consciousness, Harvard: Harvard University Press.

Grégoire, N. 2010. Identity politics, social movement and the state: 'Pan-African' associations and the making of an 'African community' in Belgium. African Diaspora, 3 (1): 159-81.

Hall, S. 1992. The Question of Cultural Identity. In: D. H. Hall et A. McGrew (éds) Modernity and Its Futures. Cambridge: Polity Press: $274-316$.

Jacobs, D., Rea, A., Teney, C., Callier, L. et Lothaire, S. 2009. L'ascenseur social reste en panne. Les performances des élèves issus de l'immigration en Communauté française et en Communauté flamande. Bruxelles: Fondation Roi Baudouin.

Jacobs, D. et Rea, A. 2011, Gaspillage de talents. Les écarts de performances dans l'enseignement secondaire entre élèves issus de l'immigration et les autres d'après l'étude PISA 2009. Bruxelles: Fondation Roi Baudouin.

Jacquemin, J.-P. (éd.) 1985. Zaïre 1885-1985: cent ans de regards belges. Bruxelles : C.E.C.

Jamoulle, P. et Mazzocchetti, J. 2011. Adolescences en exil. Paris : Academia-L'Harmattan.

Kagné, B. 200o. Africains de Belgique, de l'indigène à l'immigré. L'héritage colonial: un trou du mémoire. Hommes et Migrations, 1228: 63-64. 
Kagné, B. et Martiniello, M. 2001. L'immigration subsaharienne en Belgique. Courrier hebdomadaire du CRISP, 1721.

Kagné, B. 2008. Immigration et vie associative. Relations de l'associatif immigré subsaharien avec les pouvoirs publics. Liège : Editions Vintchore.

Lagrange, H. 200o. Reconnaissance, délinquance et violences collectives. Esprit, 268: 131-152.

Lazalo Ndoma, N. 2006. Action participativeà la citoyenneté par une communauté immigrée. L'expérience Ba YaYa. In: V. Seron et S. Smeets (éds), Participation Citoyenne et sécurité publique, enjeux émergents et expériences locales, Bruxelles : Ed. Politeia, 89-100.

Lazalo Ndoma, N. et Robert, M.-T. 2009. Les 20 ans de/à Bruxelles, plaidoyer pour la « double expertise. Agenda Interculturel, 275.

Lebovics, H. 2004. Bringing the Empire Back Home: France in the Global Age. Durham et Londres : Duke University Press.

Licata, L. et Klein, O. 2005. Regards croisés sur un passé commun : Anciens colonisés et anciens coloniaux face à l>action belge au Congo. In: M. Sanchez-Mazas et L. Licata (éds). L'Autre : Regards Psychosociaux. Grenoble : Presses Universitaires de Grenoble, 241-277.

Lusanda, N. 1993. Y a-t-il des immigrés zaïrois en Belgique? Agenda Interculturel, 112: 4-8.

Manço, U. et Kanmaz, M. 2002. De la pathologie au traitement. La gestion municipale de l'islam et des musulmans de Belgique. Cahiers d'études sur la Méditerranée orientale et le monde turcoiranien, 33: 57-88.

Manço, U. 2012a. Affaires d'identité ? Identités à faire! Travail social et «vivre ensemble ». Expériences bruxelloises. Paris : L'Harmattan.

—. 2012b. Matonge : Célébration et critique d'un quartier de la diversité par ses commerçants. In: A. Manço et C. Aschenbroich (coord.), Migrants solidaires, destin jumelés, Paris: L'Harmattan: $45^{-52}$.

Martiniello, M., Rea, A. et Dassetto, F. (éds) 2007. Immigration et intégration en Belgique Francophone. Etat des savoirs. Louvain-la-Neuve : Academia Bruylant.

Mayoyo, B. T. 1995. Migration Sud/Nord, levier ou obstacle? Les Zaïrois de Belgique. Cahiers africains, 13 .

Mazzocchetti, J. 2011. Dénis de reconnaissance, luttes et affirmation de soi. Enquête auprès de jeunes d'origine subsaharienne à Bruxelles. Uzance, 1: 88-105.

Mbembe, A. 2001. On the Postcolony, Berkeley, CA: University of California Press.

Mielants, E. 2007. Black Skin, White Masks Revisited: Contemporary Post-Colonial Dilemmas in the Netherlands, France, and Belgium. Human Architecture: Journal of the Sociology of SelfKnowledge, 5 (3): 297-304.

Mohammed, M. 2011. La formation des bandes. Entre la famille, l'école et la rue, Paris: Presses universitaires de France.

Mohammed, M. et Muchielli, L. 2007. Les bandes de jeunes, des 'Blousons noirs'à nos jours. Paris : La Découverte.

Ndaywel, I., 1998. Histoire générale du Congo. De l'héritage ancien à la République Démocratique. Paris/Bruxelles : De Boeck \& Larcier.

Ndiaye, P. 2008. La condition noire. Essai sur une minorité française. Paris : Calmann-Lévy.

Norro, P. 1990. Accueil et répartition des candidats-réfugiés politiques en Belgique. Espace, Рopulations, Sociétés, 2: 191-205.

Prévos, A. J. M. 2003. In it for Money: Rap and Business Cultures in France. Popular Music and Society, 24 (4): 445-461. 
Rea, A. 2006. Les émeutes urbaines: causes institutionnelles et absence de reconnaissance. Déviance et Société, 4 (30): 463-475.

Robert, M.-T. 2012. Bandes urbaines africaines, un produit made in Belgium. Politique (73), http:// politique.eu.org/spip.php?article2187.

Sayad, A. 1999. La double absence: des illusions de l'émigré aux souffrances de l'immigré. Paris: Seuil.

Schoonvaere, Q. \& Schoumaker, B. 2013, L'immigration subsaharienne en Belgique. État des lieux et tendances récentes, Démographie et sociétés, Document de Travail 3. Louvain-la-Neuve : Centre de recherche en démographie et sociétés.

Schoonvaere, Q. 2010. Étude de la migration congolaise et son impact sur la présence congolaise en Belgique. Analyse de données démographiques. Bruxelles: Groupe d'Étude de Démographie appliquée - Centre pour l'Égalité des Chances et de Lutte contre le Racisme.

Simon, P. 2010. 'Race', ethnicisation et discriminations : une répétition de l'histoire ou une singularité postcoloniale? In: N. Bancel et al. (éds), Ruptures postcoloniales. Les nouveaux visages de la société française. Paris : La Découverte : 357-368.

Solomos, J. 2003 (3rd edition). Race and Racism in Britain, Basingstoke: Palgrave Macmillan.

Stoler, A. L. 2008. Imperial Debris : Reflections on Ruins and Ruination. Cultural Anthropology, 23 (2): 191-219.

Swyngedouw, E. et Swyngedouw, E. 2009. The Congolese Diaspora in Brussels and Hybrid Identity Formation: multi-scalarity and diasporic Citizenship. Urban Research and Practice, 2 (1): 68-9o.

Tshibola, S., Mabaya, E. et Makobo, K. 2008. Discriminations et emploi des Africains subsahariens à Bruxelles. In: A. Manço (éd.) Valorisation des compétences et co-développement. Africain(e)s qualifié(e)s en immigration. Paris : L'Harmattan: 81-84.

Tshimanga, C. 2003. 'Belgicains', 'Parisiens' et 'Diassa-Diassa': mythes et réalités de la diaspora congolaise de retour au pays. In C. Coquery-Vidrovitch, Etre étranger et migrant en Afrique au $X X^{\text {eme }}$ siècle. Modes d'insertion et enjeux identitaires. Paris: L'Harmattan, volume 2: 491-525.

Vanderlinder, J. 2004. Le Congo dans l'imaginaire des Belges, Politique, 35.

Viaene, V., Van Reybroeck, D. et Ceuppens, B. (éds) 2009, Congo in België. Koloniale cultuur in de metropool. Leuven: universitaire Pers. 